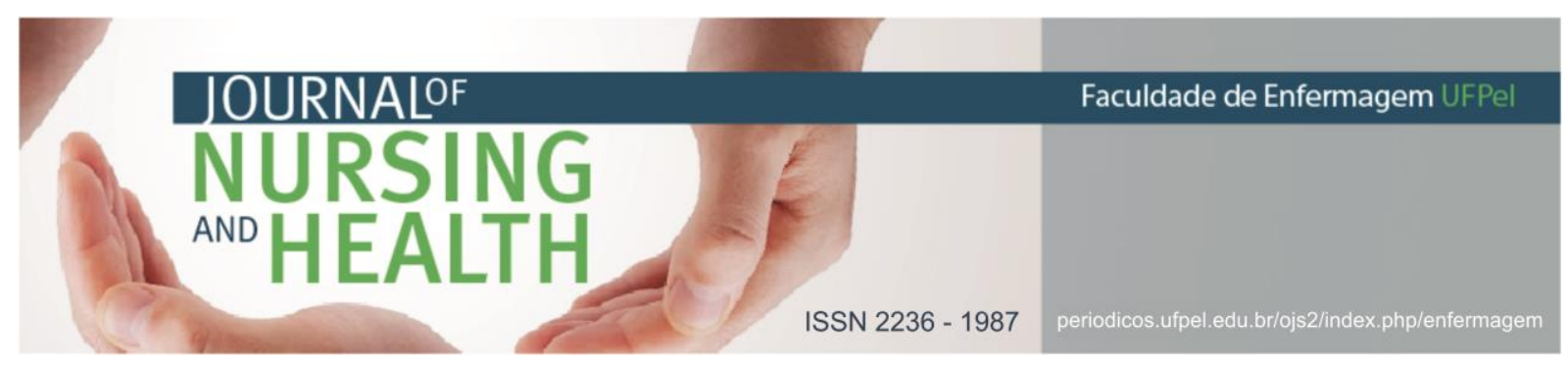

ARTIGO ORIGINAL

\title{
Oficinas terapêuticas: percepção de trabalhadores dos Centros de Atenção Psicossocial
}

\author{
Therapeutic workshops: perception of workers of the Psychosocial Care Centers \\ Talleres terapéuticos: percepción de trabajadores de los Centros de Atención
Psicosocial
}

Farias, Izamir Duarte de ${ }^{1}$; Thofehrn, Maira Buss²; Porto, Adrize Rutz ${ }^{3}$; Kantorski, Luciane Prado ${ }^{4}$

\begin{abstract}
RESUMO
Objetivo: conhecer a percepção de trabalhadores do CAPS sobre oficinas terapêuticas. Métodos: trata-se de uma pesquisa qualitativa do tipo exploratória, realizada entre abril e junho de 2013. A coleta de dados foi realizada por entrevistas semiestruturadas, da qual participaram 22 profissionais de seis Centros de Atenção Psicossocial II no município de Pelotas, Rio Grande do Sul, Brasil. A análise dos dados foi realizada a partir de uma proposta operativa. Resultados: os entrevistados mencionam falta de valorização da formação continuada ou de oportunidade para tal, muito embora, com a prática e o tempo de atuação, os trabalhadores incorporam saberes do contexto da saúde mental às suas práticas. Considerações finais: identificou-se a necessidade de conhecimento do modelo psicossocial por parte de todos os atores envolvidos no processo de trabalho do CAPS para que as oficinas sejam de fato terapêuticas e proporcionem a reabilitação psicossocial ao usuário.

Descritores: Saúde mental; Oficinas terapêuticas; Centro de atenção psicossocial.
\end{abstract}

\begin{abstract}
Objective: to know the perception of CAPS workers about therapeutic workshops. Methods: This is a qualitative research of the exploratory type, carried out from April to June of 2013. Data collection was performed by semi-structured interviews, in which 22 professionals from six Psychosocial Care Centers II participated in the city of Pelotas, Rio Grande do Sul, Brazil. Data analysis was performed based on an operational proposal. Results: interviewees mention a lack of appreciation of continuing education or the opportunity to do so, although, with practice and time, workers incorporate knowledge from the context of mental health to their practices. Final considerations: it was identified the need for knowledge of the psychosocial model by all the actors involved in the CAPS work process so that the workshops are in fact therapeutic and provide psychosocial rehabilitation to the user.
\end{abstract}

Descriptors: Mental health; Therapeutic workshops; Psychosocial care center.

\footnotetext{
${ }^{1}$ Licenciado em Artes. Doutor em Ciências da Saúde. Técnico Superior em Artes no CAPS, Pelotas, Rio Grande do Sul, Brasil. Email: izamironline@hotmail.com http://orcid.org/0000-0001-7289-8284

2 Enfermeira. Doutora em Enfermagem. Professora na Faculdade de Enfermagem da UFPel, Pelotas, Rio Grande do Sul, Brasil. Email: mairabusst@hotmail.com http://orcid.org/0000-0002-0864-3284

${ }^{3}$ Enfermeira. Doutora em Enfermagem. Professora na Faculdade de Enfermagem da UFPel, Pelotas, Rio Grande do Sul, Brasil. Email: adrizeporto@gmail.com http://orcid.org/0000-0002-5616-1626

${ }^{4}$ Enfermeira. Doutora em Enfermagem. Professora na Faculdade de Enfermagem da UFPel, Pelotas, Rio Grande do Sul, Brasil. Email: kantorski@uol.com.br http://orcid.org/0000-0001-9726-3162
} 


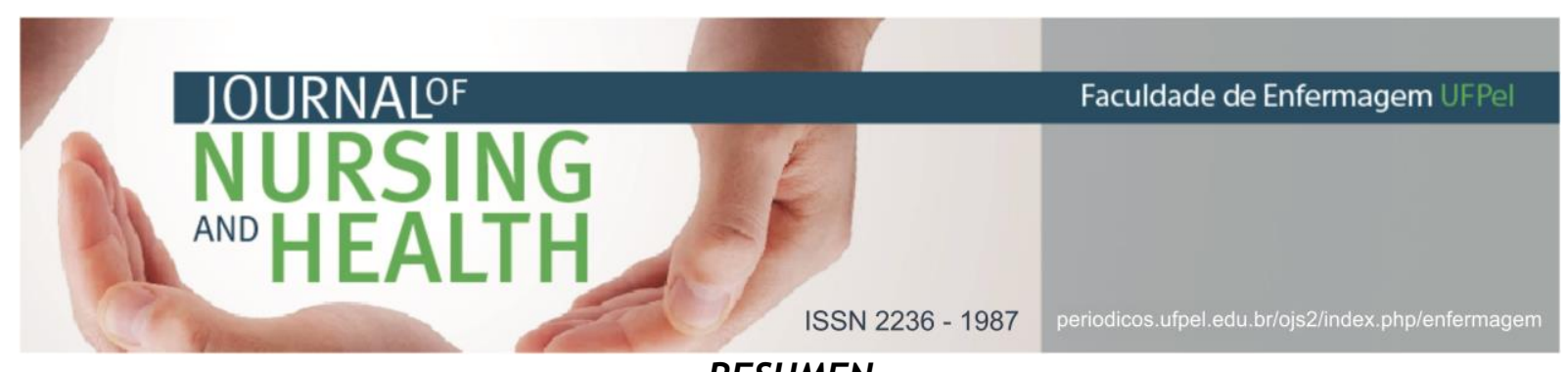

\section{RESUMEN}

Objetivo: Conocer la percepción de trabajadores del CAPS sobre talleres terapéuticos. Métodos: se trata de una investigación cualitativa del tipo exploratorio, realizada entre abril y junio de 2013. La recolección de datos fue realizada por entrevistas semiestructuradas, en la que participaron 22 profesionales de seis Centros de Atención Psicosocial II en el municipio de Pelotas, Rio Grande do Sul. El análisis de los datos se realizó a partir de una propuesta operativa. Resultados: los entrevistados mencionan falta de valorización de la formación continuada o de oportunidad para ello, aunque, con la práctica y el tiempo de actuación, los trabajadores incorporan saber del contexto de la salud mental a sus prácticas. Consideraciones finales: se identificó la necesidad de conocimiento del modelo psicosocial por parte de todos los actores involucrados en el proceso de trabajo del CAPS para que los talleres sean de hecho terapéuticos y proporcionen la rehabilitación psicosocial al usuario.

Descriptores: Salud mental; Talleres terapéuticos; Centro de atención psicosocial.

\section{INTRODUÇÃO}

A formação em saúde não está restrita aos espaços acadêmicos das universidades, pois acontece permanentemente com a prática no cotidiano de trabalho. Nos serviços de saúde mental, esta aprendizagem contínua se torna necessária ao passo que muitos profissionais tiveram sua formação acadêmica pautada no modelo asilar, regulado pela internação como único meio de tratamento. 0 rompimento com a lógica de assistência centrada na biomedicina e na doença, requer a reinvenção do fazer pelas próprias equipes de saúde a fim de destituir a ideia de loucura e investir nas potencialidades da pessoa. ${ }^{1}$

Neste sentido, o Rio Grande do Sul foi o primeiro estado brasileiro a criar a lei 9.716 em 1992 que regulamenta reforma psiquiátrica e, somente em 8 de abril de 2001 foi sancionada a lei 10.206, que passa a regulamentar os serviços substitutivos ao modelo asilar em todo Brasil. ${ }^{2}$

Com a nova política nacional de saúde mental, os Centros de Atenção Psicossocial (CAPS) foram regulamentados como serviços substitutivos ao modelo manicomial, estratégicos para concretizar o modo psicossocial de cuidado. Dentre os diferenciais do CAPS, além do cuidado em liberdade e no território do usuário, estão as oficinas terapêuticas, as quais consistem em uma ferramenta pautada na coletividade e na interação entre os sujeitos.

As oficinas terapêuticas são realizadas de acordo com as necessidades e com o interesse dos usuários, com vistas a possibilitar maior integração social e familiar, desenvolvimento de atividades produtivas, de expressão, dentre outras possibilidades. ${ }^{3}$ Ressalta-se que as oficinas em saúde mental possuem finalidades terapêuticas quando representam espaço de expressão de subjetividades permitindo acolhimento, convivência e diálogo. É sob essa perspectiva que se constituem os caminhos da reabilitação, como meio para consolidar o modo psicossocial. ${ }^{4}$

Para que estas especificidades possam ser de fato atendidas, é 


\section{JOURNALOF

fundamental o conhecimento dos profissionais que conduzem as oficinas. Pesquisas reiteram a importância da formação profissional e do trabalho em equipe para que haja compartilhamento de conhecimentos e intervenções como medidas para efetividade das oficinas terapêuticas. ${ }^{5-}$ 6

Diversos são os aspectos que requerem conhecimento técnico por parte dos profissionais, dentre os quais, trabalhar com limites e frequência nas oficinas, pode levar o coletivo da oficina a reações adversas. A organização e manejo desse aspecto exige a construção de sentido com o usuário sobre a lógica do serviço, por meio de discussões entre profissionais e definição de intervenções que julguem terapêuticas, com o intuito de avaliar o período de permanência do usuário no serviço. ${ }^{5}$

Em termos organizacionais, a oficina se torna terapêutica para o usuário quando atende suas necessidades, sendo indicada de acordo com seu perfil. Nesse sentido, a falta de entendimento de alguns profissionais sobre 0 sofrimento psíquico e a política de saúde mental dificulta a realização das oficinas ${ }^{6}$ bem como o correto encaminhamento para as mesmas.

Os trabalhadores dos CAPS que coordenam as oficinas terapêuticas devem estar motivados para buscar formação permanente e para compreenderem o trabalho em equipe como cerne deste serviço, cujo objetivo é promover a interação entre as pessoas e consequentemente à reabilitação.
Frente ao exposto, o presente estudo tem por objetivo proporcionar uma discussão sobre oficinas terapêuticas nos CAPS de Pelotas a partir da percepção de trabalhadores com formação em saúde.

\section{MATERIAIS E MÉTODOS}

Trata-se de um estudo com abordagem qualitativa, do tipo exploratório, realizado em seis CAPS II que compõem a Rede de Atenção Psicossocial no município de Pelotas, Rio Grande do Sul, Brasil.

Os dados foram coletados com 22 trabalhadores entre os meses de abril e junho de 2013 através de entrevistas semiestruturadas. Participaram quatro profissionais de cada serviço, de acordo com a formação, sendo um médico, um assistente social, um psicólogo e um enfermeiro, de maneira que essas quatro profissões de todos os CAPS acessados pudessem estar representadas nesta pesquisa.

O CAPS ADIII e o CAPS infantil não foram incluídos nesse estudo devido as suas especificidades. As entrevistas foram organizadas e analisadas conforme a proposta operativa ${ }^{7}$, pela qual os dados foram agrupados, classificados por temas e analisados, colocando-se em diálogo com autores que sustentam teoricamente as discussões.

0 anonimato dos participantes foi preservado por meio de codificação pela letra "P" seguida da ordem numérica em que as entrevistas ocorreram, assim como pela abreviatura do nome da profissão e sequência numeral dos profissionais 


\section{JOURNALOF \\ NURSING \\ aNOHEALTH}

ISSN 2236 - 1987

entrevistados da mesma área, conforme o CAPS.

Os princípios éticos para Pesquisas com Seres Humanos foram respeitados, de acordo com o previsto na Resolução $n^{\circ}$ 466/2012 do Conselho Nacional de Saúde, do Ministério da Saúde. ${ }^{8} 0$ projeto foi aprovado pelo Comitê de Ética em Pesquisa da Faculdade de Enfermagem da Universidade Federal de Pelotas, sob o parecer de $\mathrm{N}^{\circ} 232.387$, em 28 de março de 2013.

\section{RESULTADOS E DISCUSSÃO}

Quando tratamos de "credencial" em saúde, pode-se perceber 0 destaque de especialidades tradicionalmente reconhecidas, porém, a saúde mental pela lógica psicossocial requer intercâmbios entre distintos saberes e partilha de experiências cotidianas, devendo-se considerar a importância de uma gama profissional que ocupa os espaços mediadores disponibilizados pelos CAPS no Brasil.

Nesse sentido, há uma lacuna entre a formação acadêmica e as reais necessidades do Sistema Único de Saúde (SUS), uma vez que os profissionais, assim que formados, entram no mercado de trabalho e são absorvidos pelas demandas. ${ }^{9}$ Por esta razão, muitos trabalhadores não conhecem a composição da rede de serviços ou mesmo as políticas públicas que direcionam o serviço em que atuam.

Entende-se, portanto, que possibilitar aos profissionais uma formação pautada no modo psicossocial, o que é previsto em lei, é uma estratégia que busca a qualificação SUS por meio da atualização de acordo com os processos cotidianos de trabalho. ${ }^{10}$ Entretanto, muitas vezes, esta só acontecerá se houver apoio da instituição e da gestão.

Considerando as constantes transformações que vêm ocorrendo, a evolução do sistema, as novas constituições das equipes e as demandas, conjecturar oficina terapêutica pode despertar um dilema no que tange a questão: o que, prioritariamente, deve ser considerado como habilidade e competência necessárias para que um oficineiro possa desempenhar suas atividades em um CAPS?

Pela perspectiva do desenvolvimento sócio-histórico do sujeito, o aprimoramento das competências para o trabalho na oficina terapêutica, além da formação específica e da oportunidade de educação permanente, deverá ocorrer no cotidiano, por meio das relações e trocas de saberes.

A alusão a competência no âmbito profissional, refere-se à obtenção de escolaridade e aos processos de aprendizagem informais que acontecem em diferentes momentos e locais, como no próprio trabalho. ${ }^{11} \quad$ Trata-se por potencialidade, a capacidade do trabalhador para exercer suas atividades com segurança, o que é manifestado na sua prática profissional e envolve conhecimento do processo de trabalho, saberes técnicos da sua formação, sensibilidade para trabalhar com o ser humano, dentre outras. 


\section{JOURNALOF \\ NURSING

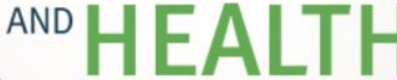

ISSN 2236 - 1987

oportunidades e de incentivo para formação, de modo que ao ingressar no CAPS, o profissional se depara com uma realidade muitas vezes completamente desconhecida e a única chance de conhecer as políticas de saúde mental, o modelo de serviço e de equipe na qual está se inserindo é com a prática cotidiana e nas suas interações com o contexto.

Outro aspecto abordado pelos participantes do estudo foi a falta da formação para que o oficineiro consiga apreender o contexto da atuação num serviço de saúde como o CAPS.

Há uma dificuldade às vezes no conhecimento, por esses profissionais não terem essa abordagem da saúde, acabam não tendo um conhecimento que poderia ajudá-los no trabalho com o usuário (P18PSI6).

Quem trabalha há mais tempo no CAPS, tem um conhecimento que falta para quem chega. Os novos profissionais precisam se capacitar um pouco nas questões da reforma psiquiátrica e nas questões de saúde, porque as vezes, algumas pessoas, chegam novas e sem esse entendimento, $e$ isso cria um problema, porque a pessoa as vezes não está para o que se objetiva no serviço, não tem a clareza do objetivo do seu trabalho. Acho que falta uma formação continuada (P6PSI12).

$\mathrm{Na}$ cidade de Pelotas conta-se com uma realidade em que os serviços integram diversos profissionais na administração das oficinas, dentre os quais, poucos possuem alguma formação em saúde. No caso dos 


\section{JOURNALOF \\ NURSING \\ AND}

ISSN 2236 - 1987

artistas, por exemplo, o campo acadêmico não contempla formação em saúde, mas estes profissionais atuam no CAPS de acordo com seu conhecimento acadêmico especifico ou suas crenças e experiências de outros modelos de serviço como palcos, ateliês ou escolas.

Muitas vezes, a arte chama atenção das pessoas pelo seu valor estético. Entretanto, as oficinas terapêuticas precisam ter o desprendimento de tal valor e dos julgamentos, para ser uma forma de criação e de expressão livre para os usuários falarem sobre si, seus afetos e seus sintomas ou potencialidades, propiciando um ambiente de elaboração de suas questões e ressignificação das suas vidas. ${ }^{13}$

O conhecimento da saúde mental e do processo de trabalho na área em que está inserido pode ser o diferencial para que os encaminhamentos desses oficineiros sejam adequados às necessidades dos usuários e efetivos enquanto recurso terapêutico, evitando assim, que a oficina seja um espaço somente produtivista.

Entende-se que as estratégias de atenção em saúde mental necessitam superar relações de competição e de hierarquização das relações sociais e produtivistas do capitalismo, valorizando além da composição de objetos, vínculos permeados por sentidos. ${ }^{14}$ Para isso, conhecer a reforma psiquiátrica e o processo de trabalho em saúde mental é fundamental para que o profissional responsável pela oficina possa tomar condutas mais assertivas, de acordo com o propósito primordial de reabilitação psicossocial.
O que tratamos como reabilitação a partir do modo psicossocial transcende a dimensão do CAPS e incide na efetiva inserção do usuário na comunidade e na sociedade. Acerca da formação profissional para desenvolver oficinas, o entrevistado aponta a importância do domínio técnico sobre a área com foco nesta reabilitação:

\section{A gente entra e tenta trazer o usuário para as práticas propostas pela oficina, e daí a importância da gente ter profissionais que sabem o que estão fazendo. Isso mostra a importância da formação profissional (P9MED1).}

Neste sentido, refere-se à formação do oficineiro, o que pode suscitar certa inquietação a respeito do modo pelo qual se compreende essa formação, pois todos os profissionais possuem formação específica para o exercício da sua prática. 0 conhecimento em questão trata-se da especificidade para a prática terapêutica em saúde mental no modo psicossocial, o que requer outro tipo de formação e sensibilidade, para que o usuário do CAPS que está fragilizado e em sofrimento possa se reabilitar.

Do ponto de vista terapêutico, pressupõe-se que haja uma posição ativa dos participantes e viabilização de compartilhamento de experiências no espaço de oficina. ${ }^{15}$ Para tanto, o oficineiro deve ser um profissional preparado para planejar e avaliar a evolução das oficinas, estando atento às manifestações e sustentando sua prática para que possa atingir os objetivos terapêuticos. As atividades 
propostas pelo serviço devem despertar o interesse do sujeito para que 0 mesmo tenha desejo de participar e possa sentir-se parte do ambiente em que está inserido.

O conhecimento, a motivação e a atitude se constituem em requisitos fundamentais para o aprimoramento da comunicação ${ }^{16}$ que consiste em um elemento fundamental para a relação entre os atores que compõem o espaço relacional da oficina. Além disso, as práticas precisam ser adequadas para o desenvolvimento das potencialidades do sujeito, sem as quais não haverá êxito na reabilitação.

Para um empreendimento eficaz dos fazeres em saúde mental, de maneira consciente e apropriada, a formação do trabalhador e 0 conhecimento das características do seu serviço são muito importantes. Esta referência diz respeito primordialmente à apropriação do saber com relação aos objetivos do CAPS enquanto instituição, e ao seu processo de trabalho, do qual 0 oficineiro é parte.

Este conhecimento pode ser adquirido basicamente de duas formas. Uma se dá por intermédio da socialização dos sujeitos no seu meio de trabalho. ${ }^{3}$ Pelo ponto de vista da construção sócio-histórica do indivíduo, o que acontece cotidianamente, ocorrem trocas de informações e experiências com profissionais mais antigos e com usuários do serviço, levando o profissional ao domínio das suas práticas. ${ }^{17}$

A outra é pela participação em eventos ou cursos para capacitação, o que requer a iniciativa da instituição (gestão pública) e o interesse do profissional para conhecer melhor as particularidades e características do seu processo de trabalho. 0 investimento da gestão em qualificação repercutirá diretamente na qualidade do cuidado prestado ao usuário.

Reiterando a ideia de que somente a formação profissional específica não dá conta das demandas do serviço, podemos perceber na fala a seguir, a relevância do trabalho em equipe e da interdisciplinaridade no contexto do CAPS, atentando-se para a maneira como os profissionais avaliam e encaminham o usuário para uma atenção ampliada:

Eu habitualmente encaminho o usuário para o assistente social ou para enfermagem, porque a pessoa, por mais psicótico, por mais doente que esteja, alguma coisa sabe fazer. Algum interesse, alguma habilidade ela tem (P15MED04).

Nesse sentido, o conhecimento da dinâmica de trabalho preconizada pelo modelo de atenção psicossocial é fundamental, muito embora, nem todos os profissionais que ingressam no CAPS possuem conhecimento desse modelo. Igualmente, tais serviços preconizam o trabalho interdisciplinar para uma atenção que contemple a singularidade do usuário num contexto de coletividade. ${ }^{18}$ Assim, a percepção da especificidade dos fazeres no contexto do CAPS é colocada em pauta:

A proposta da reforma, para o artesão, por exemplo, não é 


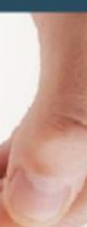

fazer um artesanato somente. É diferente de fazer em uma escola, na comunidade, numa loja. É uma proposta de saúde, então, as pessoas quando chegam, tem que ter conhecimento, saber da proposta da reforma psiquiátrica, da proposta da saúde (P6PSI2).

Às vezes, considero um pouco rígidas as oficinas e não me sinto à vontade para questionar, pois parece "invasão" ao trabalho do colega [...] a realização da tarefa proposta é mais valorizada que a presença do usuário no grupo. Penso que isso se deve à falta de conhecimento do serviço, dos objetivos em saúde mental, e também de uma formação continuada, para o profissional. Eu sei que podemos aprender no dia-a-dia, mas uma capacitação para trabalhar no CAPS, ajudaria muito (P13PSI4).

A lógica psicossocial requer conhecimentos que vão além das especificidades técnicas, ou seja, todos os espaços físicos ou relacionais do serviço devem estar disponíveis para trocas de conhecimentos e construção de novos significados. Portanto, nas salas, no refeitório, na cozinha ou em qualquer outro espaço, todos podem e devem transitar e interagir.

Entende-se que o ser humano se constrói histórico e culturalmente por meio das suas relações, sendo que, para compreendê-lo, é necessário entendermos como se dão suas relações com o meio. ${ }^{17}$ Além desta relação in loco, deve-se considerar
ISSN 2236 - 1987

locais "extra CAPS", ou seja, os espaços de vida em que o usuário transita como sua comunidade, seu bairro sua cidade, nos quais se constitui a intersetorialidade e a rede de cuidado.

$\mathrm{Na}$ relação com o outro, ocorre a internalização dos significados que representam os processos externos, de duas formas: uma refere-se ao aprendizado por meio do processamento do que já está internalizado, chamada de nível de desenvolvimento real; a outra, nível de desenvolvimento potencial, a qual se refere ao aprendizado mediado por sujeitos mais experientes por meio do convívio e das interações.

Nesta perspectiva, a construção sócio-histórica do indivíduo se dá mediante suas relações com o meio em que está inserido, ocorrendo, portanto, pelas relações e interações que o levam a compreensão do todo através da ativação da sua Zona de Desenvolvimento proximal, cujas oficinas constituem-se neste estudo como instrumento mediador ${ }^{17}$, tanto no processo de reabilitação do usuário quanto entre os profissionais.

No entanto, observam-se fragilidades e limites no universo dos CAPS, como práticas fragmentadas em ações individuais e pontuais, por cada núcleo de saber dos profissionais que compõem a equipe, e que são pouco produtoras de autonomia e cidadania. ${ }^{19}$ Compreendendo-se as oficinas como espaços que permitem a interação, um possível engessamento nas práticas por falta de formação com base no modo psicossocial causa prejuízos a esta potencialidade. 


\section{JOURNALOF \\ NURSING \\ AND}

Por outro lado, pode-se pensar na flexibilidade que o CAPS propõe para que os profissionais interajam e construam suas práticas, por meio da soma dos saberes, da interação e da consciência de que existem saberes que são tão necessários ou mais do que propriamente 0 saber técnico específico.

Ainda assim, na atualidade, as equipes são submissas ao domínio de um processo de trabalho construído ao longo da história com base na biomedicina, o qual delega atribuições a outros profissionais. ${ }^{20}$ Sabe-se que a manutenção dessa hegemonia mantém certo corporativismo dentro dos serviços e domínio sobre o coletivo, o que pode, por vezes, emperrar a evolução da reabilitação do usuário.

Assim, o significado construído para formação profissional aponta claramente para a necessidade de conhecimento do processo de trabalho em saúde mental, nos moldes da reforma psiquiátrica, o que acaba sendo apontado na fala:

[...] na verdade, cientificamente, aqui e em tudo que é CAPS, não tem ninguém com formação profissional para encaminhar uma pessoa para cá ou para lá. A assistente social não tem, o enfermeiro não tem, eu não tenho (P15MED4).

0 entrevistado refere-se à falta de formação profissional da equipe técnica do CAPS para realizar encaminhamento do usuário para as oficinas, o que pode ser considerado como uma fragilidade da equipe e, em decorrência desta, pode gerar
ISSN 2236 - 1987

insegurança ou empirismo em algumas condutas.

Em contrapartida, a cumplicidade e a partilha dos fazeres pode ser fundamental para a otimização dos recursos disponíveis no serviço, na medida em que as diversas profissões que ocupam cargos dentro dos CAPS são relevantes para tornar o cuidado mais amplo e eficiente. ${ }^{20}$

A prática interdisciplinar requer o reconhecimento de que a formação técnica para o exercício profissional no CAPS deve transcender o enfoque da escola tradicional, ampliando o conceito de saúde e considerando como conhecimento necessário e comum, aquele que tange a comunicação, a sensibilidade, a tolerância e a valorização das relações interpessoais.

\section{CONSIDERAÇÕES FINAIS}

De modo geral, o trabalho aponta para a necessidade de conhecimento do modelo psicossocial por parte de todos os atores envolvidos no processo de trabalho do CAPS, muito embora, com a prática e com o tempo de atuação estes trabalhadores vão incorporando tais saberes às suas práticas.

Mostra-se relevante o fato de que os profissionais que não possuem formação em saúde, no caso dos oficineiros, aplicam suas técnicas como se estivessem em outro espaço de trabalho, o que acaba nem sempre se alinhando com uma proposta terapêutica por conta da abordagem não levar em consideração as especificidades dos usuários. 


\section{ISSN 2236 - 1987}

Os dados apontam para o fato de que, muitas vezes, nem mesmo os profissionais com formação em saúde possuem pleno saber sobre as ações voltadas ao usuário. Deste modo é importante que haja uma avaliação e uma constante autocrítica por parte dos trabalhadores da atenção psicossocial e também no exercício de suas atividades, a fim de aprimorar suas práticas.

Cabe destacar que este estudo é recorte de um trabalho maior e que não deve ser generalizado, porém, apresenta potencial para estimular reflexões sobre o tema e novos estudos com maior profundidade, considerando outras localidades e culturas.

\section{REFERÊNCIAS}

1. Rosa RPF, Andrade ALF, Oliveira SP, Silva AGL, Ferreira AM, Inácio JS, et al. Construindo saberes no trabalho em saúde mental: experiências de formação em saúde. Interface comun saúde educ [Internet]. 2015[acesso em 2016 nov 01];19(1):931-40. Disponível em:

http: / /www.scielo.br/pdf/icse/v19s1 /1807-5762-icse-19-s1-0931.pdf

2. Ministério da Saúde (BR). Legislação em Saúde Mental 1990 - 2004 [Internet]. $5^{\mathrm{a}}$ ed. [acesso em 2017 jul 15]. Disponível em: http://portal.saude.gov.br/portal/arq uivos/pdf/Legislacao.pdf

3. Ministério da Saúde (BR). Saúde Mental no SUS: os Centros de Atenção Psicossocial [Internet]. 2004 [acesso em 2016 nov 01]. Disponível em: http://www.ccs.saude.gov.br/saude_ mental/pdf/sm_sus.html
4. Azevedo DM, Miranda FAN. Oficinas terapêuticas como instrumento de reabilitação psicossocial: percepção de familiares. Esc Anna Nery [Internet]. 2011[acesso em 2016 nov 01];15(2):339-45. Disponível em: http://www.scielo.br/pdf/ean/v15n2 /v15n2a17.pdf

5. Grigolo TM, Peres GM, Junior CAG, Rodrigues $\mathrm{J}$. $\mathrm{O}$ projeto terapêutico singular na clínica da atenção psicossocial. Cad bras saúde mental [Internet]. 2015 dez; [acesso em 2016 nov 01];7(15):53-73. Disponível em: http: / /incubadora.periodicos.ufsc.br/ index.php/cbsm/article/view/2951/4 437

6. Nunes VS, Torres MA, Zanotti SV. O psicólogo no CAPS: um estudo sobre oficinas terapêuticas. Ecos [Internet]. 2015[acesso em 2016 nov 01];5(2):35146. Disponível em: http: / /www. periodicoshumanas.uff.br /ecos/article/view/1649/1200

7. Minayo MCS. 0 desafio do conhecimento: pesquisa qualitativa em saúde. 14 ${ }^{\mathrm{a}}$ Ed. Hucitec: Rio de Janeiro; 2014.

8. Ministério da Saúde (BR). Conselho Nacional de Saúde. Resolução 466, de 12 de dezembro de 2012: diretrizes e normas regulamentadoras de pesquisa envolvendo seres humanos [Internet]. [acesso em 2016 nov 01]. Disponível em:

http://conselho.saude.gov.br/resoluc oes/2012/Reso466.pdf

9. Ministério da Saúde (BR). Programa Nacional de Reorientação da Formação Profissional em Saúde - Pró-Saúde: objetivos, implementação e desenvolvimento potencial. Brasília: Ministério da Saúde; 2007. 86 p. 
10. Vasconcelos MFF, Nicolotti CA, Silva JF, Pereira SMLR. Entre políticas (EPS - Educação Permanente em Saúde e PNH - Política Nacional de Humanização): por um modo de formar no/para o Sistema Único de Saúde (SUS). Interface comun saúde educ [Internet]. 2016[acesso em 2016 nov 01];20(59):981-91. Disponível em: http: / / www.scielo.br/pdf/icse/2016n ahead/1807-5762-icse-1807-

576220150707.pdf

11. Camelo SHH, Angerami ELS. Competência profissional: a construção de conceitos, estratégias desenvolvidas pelos serviços de saúde e implicações para a enfermagem. Texto contexto enferm [Internet]. $2013 \mathrm{abr} /$ jun[acesso em 2016 nov 01];22(2):552-60. Disponível em: http://www.redalyc.org/html/714/71 427998034/

12. Salum NC, Prado ML. A educação permanente no desenvolvimento de competências dos profissionais de enfermagem. Texto contexto enferm [Internet]. 2014 [acesso em 2016 nov 01];23(2):301-8. Disponível em: http://www.scielo.br/pdf/tce/v23n2/ pt_0104-0707-tce-23-02-00301.pdf

13. Miranda GU, Santos CF, Cunha JG, Martins TCC, Ribeiro RO, Diaz LA. Programa "Cada doido com sua mania": promoção de saúde mental e formação interdisciplinar em instituição aberta para crianças, adolescentes e adultos. Rev guará [Internet]. 2015[acesso em 2016 nov 01];1(3):93-100. Disponível em: http://periodicos.ufes.br/guara/articl e/view/9840/6662

14. Santiago E, Yasui S. Saúde mental e economia solidária: cartografias do seu discurso político. Psicol soc [Internet]. 2015[acesso em 2016 nov 01];27(3):700-11. Disponível em: http://www.scielo.br/pdf/psoc/v27n 3/1807-0310-psoc-27-03-00700.pdf

15. Baum C, Maraschin C. Oficinas e jogos eletrônicos: produção de saúde mental? Interface comun saúde educ [Internet]. 2016[acesso em 2016 nov 01];20(59):1053-1062. Disponível em: http://www.scielo.br/pdf/icse/v20n5 9/1807-5762-icse-1807-

576220180861.pdf

16. Brock D, Abu-Rish E, Chiu C, Hammer D, Wilson S, Vorvick L, et al. Interprofessional education in team communication: working together to improve patiente safety. BMJ qual saf [Internet]. 2013[accesso em 2016 nov 01];22:414-23. Disponível em: http://qualitysafety.bmj.com/conten t/22/5/414. full.pdf+html

17. Vygotsky LS. A construção do pensamento e da linguagem. $2^{\text {a }}$ ed. São Paulo: Martins Fontes; 2009.

18. Silva NS, Esperidião E, Bezerra ALQ, Cavalcante ACG, Souza ACS, Silva KKC. Percepção de enfermeiros sobre aspectos facilitadores e dificultadores de sua prática nos serviços de saúde mental. Rev bras enferm [Internet]. 2013 set/out[acesso em 2016 nov 01];66(5):745-52 Disponível em: http://www.scielo.br/pdf/reben/v66 n5/16.pdf

19. Vasconcelos MGF, Jorge MSB, Catrib AMF, Bezerra IC, Franco TB. Projeto terapêutico em Saúde Mental: práticas e processos nas dimensões constituintes da atenção psicossocial. Interface comun saúde educ [Internet]. 2016[acesso em 2016 nov 1];20(57):313-23. Disponível em: 


\section{NURSING AND}

http://www.scielo.br/pdf/icse/v20n5 7/1807-5762-icse-20-57-0313.pdf

20. Pires D. Reestruturação produtiva e trabalho em saúde no Brasil. $2^{\text {a }}$ ed. Confederação Nacional dos Trabalhadores em Seguridad Social CUT: São Paulo; 2008.

Data de submissão: 15/03/2017

Data de aceite: 10/10/2017

Data de publicação: 04/01/2018 